\title{
ON THE SCHRÖDINGER AND HEAT EQUATIONS FOR NONNEGATIVE POTENTIALS $\left({ }^{1}\right)$

\author{
BY \\ JACOB FELDMAN
}

1. Introduction. Consider the equation

$$
\frac{1}{\sigma} \frac{\partial u(x, t)}{\partial t}=(\Delta-V(x)) u(x, t)
$$

$x$ varying over Euclidean $n$-space, and $0 \leqq t<\infty$, with the initial condition $u(x, 0+)=f(x)$. For positive $\sigma$, this is the heat equation; for purely imaginary $\sigma$, it is Schrödinger's equation for a particle in a force field. In his dissertation, and later in a published article [6], R. Feynman indicated how one might get this solution as a limit of averages over polygonal paths. His prescription was not mathematically rigorous, however, since it involved infinite constants and integration with respect to a fictitious translation-invariant measure in an infinite product of real lines. In the case of the heat equation, Kac [11] made this precise by using Wiener measure instead. The approximating averages became finitedimensional approximants to a Wiener integral: for sufficiently well-behaved $V \geqq 0$ and $f$,

$$
\begin{aligned}
u(x, t) & =E\left\{\exp \left(-\int_{0}^{t} V\left(\xi_{s}+x\right) d s\right) f\left(\xi_{t}+x\right)\right\} \\
& =\lim E\left\{\exp \left[-\sum_{i} V\left(\xi_{s_{i}}+x\right) \Delta s_{i}\right] f\left(\xi_{t}+x\right)\right\}
\end{aligned}
$$

where $\xi_{t}$ is Brownian motion with parameter $\sigma$, starting at 0 , and the limit is taken as $\max \Delta s_{i} \rightarrow 0$. This was developed further by Rosenblatt [16] and Ray [15]. The problem was treated for larger classes of $V$ and for more general Markovprocesses by Getoor [8; 9], Dynkin, Volkonski1 [17], et al. Gel'fand and Yaglom [7] indicated heuristically how the same sort of approximating finite-dimensional integrals might be used to get solutions to the Schrödinger equation. They made the error of stating that, for $\operatorname{re} \sigma \neq 0$, the limit could be expressed as an integral over path space. Cameron [3] pointed out this error, but proved rigorously (for a rather narrow class of $V$ : required to satisfy certain analyticity assumptions) using certain other approximating expressions, that the limit existed for re $\sigma>0$.

Received by the editors August 13, 1962.

(1) This work was partially supported by National Science Foundation, Grant G-12911. 
The case of purely imaginary $\sigma$ was gotten as a boundary value of an analytic function. His approximants, incidentally, were not the same as those used by the nrevious authors; they corresponded to using a Simpson's rule rather than a Riemann sum to approximate $\int_{0}^{t} V\left(\xi_{s}\right) d s$. Recently, D. Babbitt, in his doctoral dissertation [1], noted that Feynman's program could be carried out rather effectively if one regarded $t$ rather than $\sigma$ as an analytic parameter. In this way, he defined a semigroup which, for $\operatorname{re} \sigma>0$, gave the solution to (1), and approximated it by an expression like (2). This worked for $V$ satisfying a local Lipschitz condition (and, again, $\geqq 0$; or, more generally, bounded below).

In the present paper we proceed as follows. First, we construct a semigroup $T_{V}^{t}$ which gives a solution to (1), for arbitrary positive measurable $V$. This was already done more generally by Getoor $[8 ; 9]$, so this is merely an exposition of a special case. Next, we investigate some smoothing properties of the operators $T_{V}^{t}$. In $\S 4$, an infinitesimal generator for $T_{V}^{t}$ is shown to exist. By means of the generator, $T_{V}^{\zeta}$ is defined for $\mathrm{re} \zeta \geqq 0$. This is then approximated by Babbitt's method, but in a more general situation. In $\S 6$, it is shown that $T_{V}^{\zeta}$ may be obtained from a Green's function, whose regularity properties are investigated.

It should be added that we have just learned that E. Nelson [14] has also succeeded in constructing a semigroup and a Feynman approximation for a large class of potentials, not necessarily bounded below.

For general background and bibliographical references, we refer the reader to $[2 ; 7 ; 12]$. The author would like to express his gratitude to D. G. Babbitt for the opportunity of seeing his manuscript at an early stage; and to E. Nelson for a stimulating discussion, and in particular, for pointing out an error in an earlier version of Theorem 3.5.

2. Brownian motion in $k$-dimensions. Let $\mathscr{K}$ be a real Hilbert space of dimension $k$, the inner product being denoted by $x \cdot y$. Let $\Omega$ be the set of continuous functions $\omega:[0, \infty) \rightarrow \mathscr{K}$, and $\xi_{t}$ the function from $\Omega$ to $\mathscr{K}$ defined by $\xi_{t}(\omega)=\omega(t)$. Let $\mathscr{F}_{t}$ be the smallest $\sigma$-field of subsets of $\Omega$ for which $\xi_{s}$ is measurable for all $s$ in $[0, t]$, and let $\mathscr{F}$ be the smallest $\sigma$-field containing all the $\mathscr{F}_{t}$.

Let

$$
G_{\sigma}(x)=(\pi \sigma)^{-k / 2} e^{-\|x\|^{2} / \sigma} \quad(\sigma>0, x \in \mathscr{K}) .
$$

Observe that $G_{\sigma}\left(x / \sqrt{ } t_{0}\right) d\left(x / \sqrt{ } t_{0}\right)=G_{t_{0} \sigma}(x) d x$. More generally, if $L_{0}$ is a nonsingular linear transformation on $\mathscr{K}$ such that $L_{0}^{*} L_{0}=t_{0} I$, then

$$
G_{\sigma}\left(L_{0} x\right) d\left(L_{0} x\right)=G_{t_{0} \sigma}(x) d x .
$$

There is a unique probability measure $\operatorname{Pr}_{x}^{\sigma}$ on $\mathscr{F}$ characterized by the property that if $h_{1}, \cdots, h_{n}>0$, and $t_{j}=h_{1}+\cdots+h_{j}$, and $S_{0}, \cdots, S_{n}$ are Borel sets in $\mathscr{K}$, then $\operatorname{Pr}_{x}^{\sigma}\left\{\xi_{0} \in S_{0}, \xi_{t_{1}} \in S_{1}, \cdots, \xi_{t_{n}} \in S_{n}\right\}=1_{S_{0}}(x) \int_{S_{1}} \cdots \int_{S_{n}} G_{h_{1}}\left(x_{1}-x\right) \cdots G_{h_{n}}\left(x_{n}-x_{n-1}\right) d x_{i} \cdots d x_{n}$.

(Notation. $1_{S}$ will mean the characteristic function of $S . I_{S}$ will mean the operation of multiplication by $1_{S}$.) 
So $\operatorname{Pr}_{x}^{\sigma}$ makes $\xi_{t}$ into a temporally homogeneous Markov process starting at $x$, with transition function

$$
\operatorname{Pr}_{x}^{\sigma}\left\{\xi_{t}=d y \mid \xi_{t_{0}}=y_{0}\right\}=G_{\sigma\left(t-t_{0}\right)}\left(y-y_{0}\right) d y .
$$

When $\sigma=1$ the superscript in $\operatorname{Pr}_{x}^{\sigma}$ will often be omitted, and when $x=0$ the subscript will often be omitted. We also denote by $E_{x}\{\cdots\}$ the operation of integration with respect to $\operatorname{Pr}_{x}^{\sigma}$, and make the same conventions about omitting $\sigma$ when it equals 1 and $x$ when it equals 0 .

Let us introduce some transformations on $\Omega$ :

(1) if $t>0$, set $j_{t}(\omega)(s)=\omega(s t)$. Thus,

$$
\xi_{s} \cdot j_{t}=\xi_{s t}
$$

(2) if $L$ is a continuous map: $\mathscr{K} \rightarrow \mathscr{K}$, we set $k_{L}(\omega)(s)=L \omega(s)$. Thus,

$$
\xi_{s}\left(k_{L}(\omega)\right)=L \xi_{s}(\omega) \text {. }
$$

REMARK 2.1. If $L y=L_{0} y-x_{0}$, where $L_{0}$ is a linear transformation on $\mathscr{K}$ with $L_{0}^{*} L_{0}=t_{0}^{-1} I$, then:

$$
\operatorname{Pr}_{x}^{\sigma} \cdot k_{L}^{-1}=\operatorname{Pr}_{L^{-1} x}^{t_{0} \sigma}
$$

In particular:

$$
\operatorname{Pr}^{\sigma} \cdot L^{-1}=\operatorname{Pr}_{x_{0}}^{t_{0} \sigma}
$$

Proof. This merely involves computing, for both sides of the equation, the measure of the set where $\xi_{0} \in S_{0}, \xi_{h_{1}} \in S_{1}, \cdots, \xi_{h_{1}+\ldots+h_{n}} \in S_{n}$, and using the transformation property of $G_{\sigma}$ observed above. The details are omitted.

3. The semigroup obtained from a potential $V$. Call a complex Borel measurable function $f$ on $\mathscr{K}$ moderate if $\int|f(x)| e^{-c\|x\|^{2}} d x<\infty$ for each $c>0$. These functions form a translation-invariant linear space $\mathscr{M}$. Observe also that a moderate $f$ is Lebesgue integrable on compact sets. For any $t>0$ and positive measurable $V$ (the value $+\infty$ being permitted), we set

$$
\begin{aligned}
T_{V}^{t} f(x) & =E_{x}\left\{\exp \left[-\int_{0}^{t} V\left(\xi_{s}\right) d s\right] f\left(\xi_{t}\right)\right\} \\
& =E\left\{\exp \left[-\int_{0}^{t} V\left(\xi_{s}+x\right) d s\right] f\left(\xi_{t}+x\right)\right\} .
\end{aligned}
$$

This makes sense, since

$$
\left|\exp \left[-\int_{0}^{t} V\left(\xi_{s}\right) d s\right] f\left(\xi_{t}\right)\right| \leqq\left|f\left(\xi_{t}\right)\right|
$$

and 
Now,

$$
E_{x}\left\{\left|f\left(\xi_{t}\right)\right|\right\} \leqq(\pi t)^{-k / 2} \int|f(y)| e^{-\|y-x\|^{2} / t} d y .
$$

$$
\frac{\|y-x\|^{2}}{t}=\frac{\|y / 2-2 x\|^{2}}{t}-\frac{3\|y\|^{2}}{4 t}+\frac{3\|x\|^{2}}{t}
$$

so

and

$$
e^{-\|y-x\|^{2} / t} \leqq e^{-3\|y\|^{2} / 4} e^{3\|x\|^{2} / t},
$$

$$
E_{x}\left\{\left|f\left(\xi_{t}\right)\right|\right\} \leqq(\pi c)^{-k / 2} e^{-3\|x\|^{2} / t} \int|f(y)| e^{-\|y\|^{2} / c} d y,
$$

where $c=(3 / 4) t$.

Measurability of the integrand is not diffficult to see, by first considering $V$ of the form $1_{s}, S$ open, and then approximating. Or see $[8 ; 9]$ for proof.

THEOREM 3.1. $T_{V}^{t}$ is a linear transformation from $\mathscr{M}$ to $\mathscr{M}$, sending a.e. nonnegative functions to nonnegative functions. If $0 \leqq f_{n} \uparrow f$ a.e., then $T_{V}^{t} f_{n} \uparrow T_{V}^{t} f$ a.e. Finally, $T_{V}^{s+t}=T_{V}^{s} T_{V}^{t}$.

Proof. All statements but the first and last are evident. To prove these:

$$
\begin{aligned}
\int\left|T_{V}^{t} f(x)\right| e^{-c\|x\|^{2}} d x & \leqq \int E_{x}\left\{\left|f\left(\xi_{t}\right)\right|\right\} e^{-c\|x\|^{2}} d x \\
& =\int G_{t} *|f|(x) e^{-c\|x\|^{2}} d x \quad \text { (where * is convolution) } \\
& =\left(\frac{\pi}{c}\right)^{k / 2} \int|f(x)| G_{t} * G_{1 / c}(x) d x \\
& =\left(\frac{\pi}{c}\right)^{k / 2} \int|f(x)| G_{t+1 / c}(x) d x<\infty .
\end{aligned}
$$

So $T_{V}^{t}$ takes $\mathscr{M}$ to $\mathscr{M}$. Finally:

$$
\begin{aligned}
T_{V}^{s} T_{V}^{t} f(x) & =E_{x}\left\{\exp \left[-\int_{0}^{s} V\left(\xi_{r}\right) d r\right] T_{V}^{t} f\left(\xi_{s}\right)\right\} \\
& =E_{x}\left\{\exp \left[-\int_{0}^{s} V\left(\xi_{r}\right) d r\right] E_{\xi_{s}}\left\{\exp \left[-\int_{0}^{t} V\left(\xi_{u}\right) d u\right] f\left(\xi_{t}\right)\right\}\right\} \\
& =E_{x}\left\{\exp \left[-\int_{0}^{s} V\left(\xi_{r}\right) d r\right] \exp \left[-\int_{0}^{t} V\left(\xi_{u+s}\right) d s\right] f\left(\xi_{t+s}\right)\right\}
\end{aligned}
$$

by the strong Markov property. This can be rewritten as $T_{V}^{s+t} f(x)$.

Next, we examine the effect of varying $V$.

THEOREM 3.2. $V \geqq W$ a.e. $\Rightarrow T_{V}^{t} f \leqq T_{W}^{t} f$ for all a.e. nonnegative $f$ in $\mathscr{M}$. Furthermore, if $V_{n} \uparrow V$ a.e., then $T_{V_{n}}^{t} f \downarrow T_{V}^{t} f$ for such $f$. 
Proof. The only difficulty is to see that if $V=W$ a.e., then $T_{V}^{t} f=T_{W}^{t} f$. This is true because, for any set $S$ of measure 0 in $\mathscr{K}$, we have

$$
E_{x}\left\{\int_{0}^{t} 1_{s}\left(\xi_{s}\right) d s\right\}=\int_{0}^{t} \int_{S} G_{x}(y-x) d y d s=0
$$

so that $V$ is unaffected by a change on a set of measure 0 .

Finally, we consider the action of $T_{V}^{t}$ on the various $\mathscr{L}_{p}$ spaces over $\mathscr{K}$ (taken with Lebesgue measure, normalized via the inner product in $\mathscr{K}$ ), and also on the space $\mathscr{B}$ of bounded Borel functions on $\mathscr{K}$. By $\|\cdot\|_{p}$ we will mean the norms in $\mathscr{L}_{p}=\mathscr{L}_{p}(\mathscr{K}), 1 \leqq p \leqq \infty$, and by just plain $\|\cdot\|$ the norm in $\mathscr{B}$.

THEOREM 3.3. $\quad T_{V}^{t}$ is a contraction on $\mathscr{L}_{p}(1 \leqq p \leqq \infty)$ and on $\mathscr{B}$. Furthermore, if $f \in \mathscr{L}_{p}$ and $g \in \mathscr{L}_{q}$, with $1 / p+1 / q=1$, then $\int \bar{T}_{V}^{t} f(x) g(x) d x=\int f(x) T_{V}^{t} g(x) d x$.

Proof. Since $T_{V}^{t} f(x) \leqq T_{0}^{t} f(x)$ for each nonnegative $f$ in $\mathscr{M}$, the first sentence will follow once we have it for the case $V=0$. But this case is well known for $p=1$ and $p=\infty$, while for other $p$ it follows from the Riesz convexity theorem.

As for the self-adjointness: Consider $\Omega \times \Omega$. If we denote by $\tilde{\Omega}$ the subset of pairs $\left(\omega, \omega^{\prime}\right)$ such that $\xi_{0}(\omega)=\xi_{0}\left(\omega^{\prime}\right)$, then $\tilde{\Omega}$ can be identified with the space of all continuous functions $\tilde{\omega}$ from the real line to $\mathscr{K}$, by letting

$$
\tilde{\omega}(t)= \begin{cases}\omega(-t) & \text { if } t \leqq 0 \\ \omega^{\prime}(t) & \text { if } t \geqq 0\end{cases}
$$

The measure $\operatorname{Pr}_{x} \times \operatorname{Pr}_{x}$ has its support on $\tilde{\Omega}$. We set $\tilde{\operatorname{Pr}}\{\tilde{\Lambda}\}=\int \operatorname{Pr}_{x} \times \operatorname{Pr}_{x}\{\tilde{\Lambda}\} d x$, for $\tilde{\Lambda}$ a measurable set in $\tilde{\Omega}$. $\tilde{P} r$ is, of course, an infinite measure. Define $\tilde{\xi}_{t}(\tilde{\omega})=\tilde{\omega}(t)$. Then it is easy to see that the joint distributions of $\tilde{\xi}_{t_{1}}, \cdots, \tilde{\xi}_{t_{n}}$ are the same as those of $\tilde{\xi}_{t_{1}+h}, \cdots, \tilde{\xi}_{t_{n}+h}$, and of $\tilde{\xi}_{-t_{1}}, \cdots, \tilde{\xi}_{-t_{n}}$. Thus,

$$
\begin{aligned}
\left(T_{V}^{t} f, g\right) & =\int E_{x}\left\{\exp \left[-\int_{0}^{t} V\left(\xi_{s}\right) d s\right] f\left(\xi_{t}\right)\right\} g(x) d x \\
& =\tilde{E}\left\{\exp \left[-\int_{0}^{t} V\left(\tilde{\xi}_{s}\right) d s\right] f\left(\tilde{\xi}_{t}\right) g\left(\tilde{\xi}_{0}\right)\right\}=\tilde{E}\left\{\exp \left[-\int_{0}^{t} V\left(\tilde{\xi}_{t-s}\right) d s\right] f\left(\tilde{\xi}_{0}\right) g\left(\tilde{\xi}_{t}\right)\right\} \\
& =E\left\{\exp \left[-\int_{0}^{t} V\left(\tilde{\xi}_{u}\right) d u\right] f\left(\tilde{\xi}_{0}\right) g\left(\tilde{\xi}_{t}\right)\right\}=\int E_{x}\left\{\exp \left[-\int_{0}^{t} V\left(\xi_{u}\right) d u\right] g\left(\xi_{t}\right)\right\} f(x) d x \\
& =\left(f, T_{V}^{t} g\right) .
\end{aligned}
$$

REMARK. The self-adjointness has been proved by Getoor for $\mathscr{L}_{2}$, in [8], and could be shown generally by approximation.

THEOREM 3.4. If $f$ is in $\mathscr{L}_{p}$ and $1 / q+1 / p=1$, then $\left\|T_{V}^{t} f\right\|<C(q) t^{l(q)}\|f\|_{p}$, where $C(q)=\pi^{l(q)} q^{-k / 2 q}$ and $l(q)=(1 / q-1) k / 2$. Furthermore, if $S_{N}$ is the 
$N$-sphere, then $\left\|I_{S_{N}} T_{V}^{t} f\right\| \rightarrow 0$ as $N \rightarrow \infty$, uniformly in $V$ and in $t$ restricted to an interval $0<t_{0}<t<t_{1}<\infty$.

Notation. $S^{\perp}$ is the complement of the set $S$.

Proof. $\left|T_{V}^{t} f(x)\right| \leqq\left|G_{t} * f(x)\right| \leqq\left\|G_{t}\right\|_{q}\|f\|_{p}$. Evaluating $\left\|G_{t}\right\|_{q}$ gives the first part.

Choose $\varepsilon>0$. Choose $M$ so big that $\left\|I_{S_{M}} f\right\|_{p}<\varepsilon\left(2\left\|G_{t_{0}}\right\|_{q}\right)^{-1}$. Then $\left\|T_{V}^{t} I_{S_{M}} f\right\|<\varepsilon / 2$. Now, if $\|y\| \leqq M$ and $\|x\| \geqq N$, then $\|y-x\| \geqq N-M$, and $\left|G_{t}(y-x)\right| \leqq\left(\pi t_{0}\right)^{-k / 2} e^{-(N-M)^{2} / t_{1}}$ provided $t_{0} \leqq t \leqq t_{1}$. Now, $I_{S_{M}} f$ is an $\mathscr{L}_{p}$ function with support of finite measure, hence an $\mathscr{L}_{1}$ function. Choose $N$ so large that $\left|G_{t}(y-x)\right|<\varepsilon\left(2\left\|I_{S_{M}} f\right\|_{1}\right)^{-1}$ if $\|y\| \leqq M,\|x\| \geqq N$, and $t_{0} \leqq t \leqq t_{1}$. Then $\left|T_{V}^{t} f(x)\right|<\varepsilon$ if $\|x\| \geqq N$ and $t_{0} \leqq t \leqq t_{1}$.

LEMMA. If $V_{0}$ is in $\mathscr{L}_{p}$ for some $p>k / 2$, then

Proof.

$$
E_{x}\left\{\int_{0}^{r} V_{0}\left(\xi_{u}\right) d u\right\} \leqq c(p)\left\|V_{0}\right\|_{p} \cdot r^{k / 2+1} .
$$

$$
\begin{aligned}
\left|E_{x}\left\{\int_{0}^{r} V_{0}\left(\xi_{u}\right) d u\right\}\right| \mid & =\int_{0}^{r} V_{0}(x+y) G_{u}(y) d y d u \mid \\
& \leqq \int_{0}^{r}\left\|V_{0}\right\|_{p}\left\|G_{u}\right\|_{q} d u=\left\|V_{0}\right\|_{p} \int_{0}^{r} C(q) u^{l(q)} d u
\end{aligned}
$$

(using the notation in the proof of the previous theorem)

$$
=c(p)\left\|V_{0}\right\|_{p} r^{k / 2+1}
$$

where $c(p)=C\left((1-1 / p)^{-1}\right)(1-k / 2 p)^{-1}$.

TheOREM 3.5. If, for some $\bar{p}>k / 2, V$ is in $\mathscr{L}_{\bar{p}}$ on an open set $\mathcal{O}$, and $f$ is in any $\mathscr{L}_{p}$ class, then $T_{V}^{t} f$ is continuous in $\mathcal{O}$. More precisely: for any compact subset $C$ of $\mathcal{O}, \varepsilon>0$, and $t_{1}>0$, we can choose $r_{0}$ such that $T_{V}^{t} f(x)$ differs by less than $\varepsilon$ from the continuous function $T_{0}^{r} T_{V}^{t-r} f(x)=G_{r} * T_{V}^{t-r} f(x)$, for all $x$ in $C, 0<r \leqq r_{0}$, and $t \geqq t_{1}$.

Proof. Let $\Lambda_{s}=\left\{\omega \mid \xi_{r}(\omega) \in \mathcal{O}\right.$ for $\left.0 \leqq r \leqq s\right\}$. This is in $\mathscr{F}$, as is also the set $\Gamma_{s, \mathrm{z}}=\left\{\omega \mid \int_{0}^{s} V\left(\xi_{r}\right) d r<\alpha\right\}$. Write $\Phi_{r}^{s}$ for the function $\exp \left[-\int_{r}^{s} V\left(\xi_{u}\right) d u\right]$ on $\Omega$. Then

$$
\begin{aligned}
T_{V}^{t} f(x)=E_{x}\left\{\Phi_{0}^{t} f\left(\xi_{t}\right)\right\}= & E_{x}\left\{\Lambda_{s}^{\perp} \cup \Gamma_{r, \alpha}^{\perp},\left(\Phi_{0}^{r}-1\right) \Phi_{r}^{t} f\left(\xi_{t}\right)\right\} \\
& +E_{x}\left\{\Lambda_{s} \cap \Gamma_{r, \alpha},\left(\Phi_{0}^{r}-1\right) \Phi_{r}^{t} f\left(\xi_{t}\right)\right\}+T_{0}^{r} T_{V}^{t-r} f(x)
\end{aligned}
$$

We estimate the first two terms. 
Let $C$ be a compact subset of $\mathcal{O}, d$ its distance from $\mathcal{O}^{\perp}$, and $x$ any point in $C$. Then $\operatorname{Pr}_{x}\left\{\Lambda_{s}^{\perp}\right\} \leqq \operatorname{Pr}_{0}\left\{\left\|\xi_{u}\right\| \geqq d\right.$ for some $u$ in $\left.[0, s]\right\}$. This is known to go to 0 as $s \downarrow 0$. Thus, we can choose $s$ so small that $\operatorname{Pr}_{x}\left\{\Lambda_{s}^{\perp}\right\} C(q)\left(t_{1} / 2\right)^{l(q)}<\varepsilon / 3(l(q)$ defined as in Theorem 3.4). We can also require that $s<t_{1} / 2$.

Next, denote by $V_{0}$ the function $I_{\mathcal{O}} V$. Then if $r \leqq s$,

$$
\operatorname{Pr}_{x}\left\{\Lambda_{s} \cap \Gamma_{\alpha, r}^{\perp}\right\}=\operatorname{Pr}_{x}\left\{\Lambda_{s}, \int_{0}^{r} V_{0}\left(\xi_{u}\right) d u \geqq \alpha\right\} \leqq \operatorname{Pr}_{x}\left\{\int_{0}^{r} V_{0}\left(\xi_{u}\right) d u \geqq \alpha\right\} .
$$

From the previous lemma, this is dominated by $c(\bar{p}) / \alpha\left\|V_{0}\right\|_{p^{r^{\prime}}}^{-k+1}$. If $\alpha$ is preassigned, then by choosing $r$ sufficiently small (and in particular, smaller than $s$ and $t_{1} / 2$ ), we can thus guarantee that

$$
\operatorname{Pr}_{x}\left\{\Lambda_{s} \cap \Gamma_{c, r}^{\perp}\right\} C(q)\left(t_{1} / 2\right)^{l(q)}<\varepsilon / 3 .
$$

$\alpha$ was at our disposal. We choose it so small that $\left(1-e^{-\alpha}\right) C(q)\left(t_{1} / 2\right)^{l(q)}<\varepsilon / 3$. Then

$$
\begin{aligned}
\left|E_{x}\left\{\Lambda_{s} \cap \Gamma_{r, \alpha}\left(\Phi_{0}^{r}-1\right) \Phi_{r}^{t} f\left(\xi_{t}\right)\right\}\right| & \leqq\left(1-e^{-\alpha}\right) E_{x}\left\{\Phi_{r}^{t}|f|\left(\xi_{t}\right)\right\} \leqq\left(1-e^{-\alpha}\right) E_{x}\left\{T_{V}^{t-r}|f|\left(\xi_{r}\right)\right\} \\
& \leqq\left(1-e^{-\alpha}\right) C(q)(t-r / 2)^{l(q)}\|f\|_{p} \leqq \varepsilon / 3\|f\|_{p}
\end{aligned}
$$

Thus, for all $t \geqq t_{1}$ and $x$ in $C,\left\|T_{V}^{t} f-T_{0}^{r} T_{V}^{t-r} f\right\|$ is dominated by $\varepsilon\|f\|_{p}$, the choice of $r$ depending on $\varepsilon, t_{1}$ and $\left\|V_{0}\right\|_{\bar{p}}$.

Summarizing some of this:

Corollary 3.1. $T_{V}^{t}$ takes each $\mathscr{L}_{p}$ class into $\mathscr{L}_{p} \cap \mathscr{B}_{0}$ (where $\mathscr{B}_{0}$ is $\{f$ in $\mathscr{B} \mid f(x) \rightarrow 0$ as $\|x\| \rightarrow \infty\})$. Furthermore, $T_{V}^{t} f$ is continuous on the open set $\left\{x \mid V\right.$ is in $\mathscr{L}_{p}$ in some neighborhood of $x$, for some $\left.p>k / 2\right\}$.

REMARK 3.1. Choosing $V$ to be $+\infty$ on the complement of some set is one way of relativizing the process to that set (see also the method used by Getoor in [9], where everything gets cut down to an open set $G$ ).

4. The infinitesimal generator. In [8] Getoor incorrectly said that $T_{V}^{t}$ is continuous at 0 as a semigroup on $\mathscr{L}_{2}$ (and hence, has a densely defined infinitesimal generator). This statement was, however, corrected in [9], and even in [8] he mentioned a necessary and sufficient condition on $V$ that $T_{V}^{t}$ be continuous in this sense. Also, in [9], a rather stringent sufficient condition is given. The contion in [8] is just that $\lim _{t \rightarrow 0} \exp \left[-\int_{0}^{t} V\left(\xi_{s}\right) d s\right]=1 \operatorname{Pr}_{x}-$ a.e., for almost every $x$ in $\mathscr{K}$. We shall not assume this, but rather investigate for arbitrary $V$ the subspace on which $T_{V}^{t}$ is continuous; or, equivalently, the closure of the domain of the infinitesimal generator of $T_{V}^{t}$.

Consider, for fixed $x$, the condition that $\lim _{t \rightarrow 0} \exp \left[-\int_{0}^{t} V\left(\xi_{s}\right) d s\right]=0 \operatorname{Pr}_{x}^{\sigma}-$ a.e. This condition is actually independent of $\sigma$. One way of seeing this is the following. Recall the map $j_{\sigma}$ from $\Omega$ to $\Omega$ sending $\omega$ to the function whose value at 
$t$ is $\omega(t \sigma)$. Then $j_{\sigma}$ is a $1-1$ measure-preserving transformation from $(\Omega, \mathscr{F}, \operatorname{Pr})$ to $\left(\Omega, \mathscr{F}, \operatorname{Pr}^{\sigma}\right)$, and $\int_{0}^{t \sigma} V\left(\xi_{s}+x\right) d s=\sigma \int_{0}^{t} V\left(\xi_{s} \cdot j_{\sigma}+x\right) d s$.

Let $\Omega_{x}=\left\{\lim _{t \rightarrow 0} \exp \left[-\int_{0}^{t} V\left(\xi_{s}+x\right) d s\right]=1\right\}$. This is exactly

$$
\left\{\lim _{t \rightarrow 0} \int_{0}^{t} V\left(\xi_{s}+x\right) d s=0\right\}
$$

and also $\left\{\exists t>0\right.$ such that $\left.\int_{0}^{t} V\left(\xi_{s}+x\right) d s<\infty\right\}$. The set is in $\mathscr{F}_{t}$ for each $t>0$. Thus, by the zero-one law, it differs from a set in $\mathscr{F}_{0}$ by a set which has $\operatorname{Pr}^{\sigma}$-measure 0 for each $\sigma$. Then for given $x, \Omega_{x}$ either has $\operatorname{Pr}^{\sigma}$-measure 0 for all $\sigma$ or has $\operatorname{Pr}^{\sigma}$-measure 1 for all $\sigma$.

$V$ will be called controllable at $x$ if $\operatorname{Pr}\left\{\Omega_{x}\right\}=1$. So if $V$ is not controllable at $x$, then for each moderate $f$ we have $T_{V}^{t} f(x)=0$ for all $t>0$.

Definition. Let $C_{V}$ be the set of points where $V$ is controllable, and let $I_{V}$ be the operation of multiplication by the function which is 1 on $C_{V}$ and 0 elsewhere. Also, let $\mathscr{L}_{p}^{V}$ be $\mathscr{L}_{p}$ with respect to Lebesgue measure cut down to $C_{V}$, with corresponding norms \|\|$_{p}^{V}$.

THEOREM 4.1. (a) For each moderate $f$ we have

$$
I_{V} T_{V}^{t} I_{V} f=T_{V}^{t} f \text { a.e., }
$$

and

$$
\lim _{t \downarrow 0} T_{V}^{t} f=I_{V} f \text { a.e. }
$$

(b) Furthermore, $T_{V}^{t}$ is strongly continuous on each $\mathscr{L}_{p}^{V}, 1 \leqq p<\infty$, and is weak * continuous on $\mathscr{L}_{\infty}^{V}$. Let $A_{V}$ be the infinitesimal generator (no distinction is necessary for different $p$, since there is agreement on functions in several different $\mathscr{L}_{p}^{V}$ ).

(c) On $\mathscr{L}_{2}^{V}, A_{V}$ is a nonnegative self-adjoint operator, and $e^{-t A_{V}}=T_{V}^{t}$.

Proof. If $x$ is not in $C_{V}$, then $E\left\{\exp \left[-\int_{0}^{t} V\left(\xi_{s}+x\right) d s\right] f\left(\xi_{t}+x\right)\right\}=0$ for each $t>0$. So $I_{V} T_{t} f={ }_{\text {a.e. }} T_{V}^{t} f$ for moderate $f$. If $f$ is actually in $\mathscr{L}_{2}$, then selfadjointness of $T_{V}^{t}$ tells us that $I_{V} T_{V}^{t} I_{V} f={ }_{\text {a.e. }} T_{V}^{t} f$. For general moderate $f$, the last equality still holds, by the Lebesgue convergence theorem. The fact that $\lim _{t \downarrow 0} T_{V}^{t} f(x)=f(x)$ for a.e. $x$ in $C_{V}$ can be shown as follows: first one proves it for continuous $f$, by applying the Lebesgue convergence theorem; then for arbitrary moderate $f$ by Theorem 3.1 .

As for (b): since $T_{V}^{t} f$ converges a.e. to $I_{V} f$ as $t \downarrow 0$, and since $\left\|T_{V}^{t} f\right\|_{p}^{V} \leqq\left\|T_{V}^{t} f\right\|_{p}=\left\|T_{V}^{t} I_{V} f\right\|_{p} \leqq\left\|I_{V} f\right\|_{p}=\|f\|_{p}^{V}$, we have that $T_{V}^{t} f \rightarrow f$ in $\mathscr{L}_{p}^{V}$, in the weak topology, or, in the case $p=\infty$ in the weak * topology. Since each $T_{V}^{t}$ is a contraction, we get $T_{V}^{t}$ weakly continuous at all $t \geqq 0$ if $1 \leqq p<\infty$, or weak * continuous if $p=\infty$. If $1 \leqq p<\infty$, then $\mathscr{L}_{p}^{V}$ is separable, so weak continuity implies strong measurability by [10], Theorem 3.55 , and therefore $T_{V}^{t}$ is strongly continuous for $t \geqq 0$ by [10, Theorem 10.5 .5$]$. 
(c) Finally, that $A_{V}$ on $\mathscr{L}_{2}^{V}$ is a nonnegative self-adjoint operator can be seen as follows. $T_{V}^{t}$ has some representation of the form $e^{-t B}$ for a self-adjoint operator $B$ (easily seen to be positive), by [13, XI,2]. Now, the domain of $A_{V}$ is the range of $\int_{0}^{\infty} T_{V}^{t} e^{-\lambda t} d t=1 /(B+\lambda)$, and $\left(A_{V}+\lambda\right)(1 /(B+\lambda)) f=f$. Thus, $A_{V}$ is precisely $B$.

Next, two theorems which cast a little light on the question "what is $C_{V}$ for given $V$ '?

THEOREM 4.2. $C_{V}$ is a.e. contained in the set where $V$ is finite.

Proof. Let $R=\{x \mid V(x)=\infty\}$. Then, for a.e. $x$ in $R$, the set $R$ has density 1 at $x$. Selecting such an $x$ :

$$
E_{x}\left\{\frac{1}{t} \int_{0}^{t} 1_{R}\left(\xi_{s}\right) d s\right\}=\frac{1}{t} \int_{0}^{t} E_{x}\left\{1_{R}\left(\xi_{s}\right)\right\} d s=\frac{1}{t} \int_{0}^{t} G_{R} * 1_{R}(x) d s .
$$

Now, $G_{s} * 1_{R}(x) \rightarrow 1$ as $s \rightarrow 0$, since $G_{s}$ is an approximate identity and $R$ has density 1 at $x$. Thus

$$
\frac{1}{t} \int_{0}^{t} G_{s} * 1_{R}(x) d s \rightarrow 1 .
$$

As a consequence, $\operatorname{Pr}_{x}\left\{\int_{0}^{t} V\left(\xi_{s}\right) d s=\infty\right\}=1$ for each $t>0$, and $x$ is in $R$.

THEOREM 4.3. If $p>k / 2$ and $V \in \mathscr{L}_{p}(\mathcal{O})$, $\mathcal{O}$, open $\subset K$, then $C_{V} \supset \mathcal{O}$ a.e.

Proof. It is no loss of generality to assume $V$ vanishes outside $\mathcal{O}$, since each path starting at an $x$ in $\mathcal{O}$ stays there for a while. But then the lemma after Theorem 3.4 tells us that $E_{x}\left\{\int_{0}^{t} V\left(\xi_{s}\right) d s\right\}<\infty$, so that $\int_{0}^{t} V\left(\xi_{s}\right) d s<\infty \operatorname{Pr}_{x}$-a.e., and therefore $x$ is in $C_{V}$ if it is in $\mathcal{O}$.

REMARK 4.1. Operators on $\mathscr{L}_{p}^{V}$ are in an obvious 1-1 correspondence with operators $B$ on $\mathscr{L}_{p}$ such that $I_{V} B I_{V}=B$. Thus, we will occasionally treat $A_{V}$ as an operator on $\mathscr{L}_{p}$, without further comment.

REMARK 4.2. If $V=0$, then $A_{V}$ is just the negative of the usual Laplacian (on $\mathscr{L}_{2}$ ). More generally, it is shown in [8] that if $C_{V}$ is almost all of $\mathscr{K}$, and $M_{V}$ is the operation of multiplication by $V$ on $\mathscr{H}$, then

$$
\left.A_{V} \supset\left(-\Delta+M_{V}\right) \mid \mathscr{D}_{\Delta} \cap \mathscr{D}_{M_{V}} \quad \text { (where } \mathscr{D}_{T} \text { is the domain of } T\right) .
$$

For example, if $V$ is bounded, then $A_{V}$ is just $-\Delta+M_{V}$. However, it would be of interest to have the answer to the following question, for instance. Suppose the Laplacian of $f$ exists locally, in some sense, but the function $g$ thereby obtained is no longer in $\mathscr{L}_{2}$. Suppose, however, that $-g+V f$ is in $\mathscr{L}_{2}$. Is it then the case that $f$ is in $\mathscr{D}_{A_{V}}$ and $A_{V} f=-g+V f$ ? (The considerations of $[9, \S 4]$, do not apply, unfortunately, because $\Delta$ is not a "local operator" in the sense used there.) 
REMARK 4.3. Here is a phenomenon which was surprising at least to me. Recall that

$$
T_{0}^{t}=e^{-t \Delta} f=G_{t} * f
$$

is actually infinitely differentiable for all moderate $f$. Recall also that for general $V$, and $f$ in $\mathscr{L}_{p}, i T_{V}^{t}$ is continuous where $V$ is in some $\mathscr{L}_{\bar{p}}$ class (Corollary 3.1). One might therefore expect that if, say, $V$ were bounded then $T_{V}^{t} f$ would be infinitely differentiable. However, this is far from true!

EXAMPLE. Let $V$ be a nonnegative bounded measurable function, and let $f$ be in $\mathscr{D}_{A_{V}}$ Thus $T_{V}^{t} f$ is again in $\mathscr{D}_{A_{V}}$, and $A_{V} T_{V}^{t} f=T_{V}{ }^{t} A_{V} f$. But $\mathscr{D}_{A_{V}}=\mathscr{D}$ and $A_{V}=-\Delta+M_{V}$, so $-\Delta T_{V}^{t} f+V T_{V}^{t} f=A_{V} T_{V}^{t} f=T_{V}^{t} A_{V} f$. Now, $T_{V}^{t} f$ is continuous, as is also $T_{V}^{t} A_{V} f$, by Corollary 3.1. If $T_{V}^{t} f$ had two continuous derivatives, then a representative for $T_{V}^{t} f$ could be chosen which was continuous. But $V T_{V}^{t} f$ can be made as irregular as one likes, for example by choosing $V$ to be unequal to any continuous function on any open set. So $T_{V}^{j} f$ cannot always be twice continuously differentiable, even if $V$ is bounded and $f$ itself is a $C_{\infty}$ function with compact support. (However, regularity assumptions on $V$ would presumably result in regularity for $T_{V}^{t} f$.)

5. Complexification of the semigroup, and the limiting Feynman integral. Let $\Lambda$ be the set of complex numbers with positive real part, $\bar{\Lambda}$ its closure. Let $A$ be a fixed nonnegative self-adjoint operator on the Hilbert space $\mathscr{H}$. For any $\zeta$ in $\bar{\Lambda}$, the functional calculus defines a bounded operator $e^{-\zeta A}$. This operator is unitary if $\zeta$ is imaginary, nonnegative and self-adjoint if $\zeta$ is nonnegative, and has norm $\leqq 1$ for $\zeta$ in $\bar{\Lambda}$. The map $\zeta \rightarrow e^{-\zeta A}$ is continuous in the strong operator topology for $\zeta$ in $\bar{\Lambda}$, and satisfies $e^{-\zeta A} e^{-\zeta^{\prime} A}=e^{-\left(\zeta+\zeta^{\prime}\right) A}$. For $\zeta$ in $\Lambda$,it is continuous in the uniform operator topology, and even holomorphic. These facts are all, at worst, straightforward applications of the functional calculus.

EXAMPLE. We can extend $T_{V}^{t}$, as an operator on $\mathscr{L}_{2}^{V}$, to $T_{V}^{\zeta}$ for $s$ in $\bar{\Lambda}$, by setting $A=A_{V}$.

We quote, for later use, a fact abo'st convergense of analytic functions.

FACT 5.1 (Vitali). Let $F_{1}, F_{2}, \cdots$ be a sequence of analytic functions on $\Lambda$, with values in a Banach space. Suppose the $F_{n}$ are uniformly bounded in norm on each compact subset of $\Lambda$. Suppose also that they converge in norm at all points of $(0, \infty)$. Then they converge in norm on $\Lambda$, uniformly on compact subsets, to an analytic function $F_{\infty}$ on $\Lambda$.

Proof. $[10$, p. 104, Theorem 3.14.1].

For the purposes of our first theorem, we will want, for each $t>0$, $\operatorname{Pr}_{x}\left\{V\left(\xi_{s}\right)\right.$ Riemann-integrable on $\left.(0, t)\right\}=1$ for a.e. $x$. This amounts to $\operatorname{Pr}_{x}\left\{V\left(\xi_{s}\right)\right.$ bounded and a.e. continuous on $\left.(0, t)\right\}=1$ for a.e. $x$. Call such a $V$ Riemann-approximable. Observe that if $V$ is Reimann-approximable then $C_{V}$ is almost all of $E$, so that $T_{V}^{t}$ is strongly continuous at 0 in $\mathscr{L}_{p}$. 
THEOREM 5.1. Suppose Vis Riemann-approximable. Let $\tau$ be a finite sequence of positive numbers: $\tau=\left(\tau_{1}, \cdots, \tau_{n(\tau)}\right)$, with $\sum \tau_{j}=1$. Let $|\tau|=\max _{j} \tau_{j}$. For $\zeta$ in $\Lambda$, let $T_{V, \tau}^{\zeta}=\prod_{j} e^{-\zeta \tau_{j} V} e^{\zeta \tau_{j} \Delta}$, everything operating on $\mathscr{L}_{2}$. This is clearly holomorphic on $\Lambda$, strongly continuous on $\bar{\Lambda}$. Then $\lim _{|\tau| \rightarrow 0} T_{V, \tau}^{\zeta}$ exists in the strong operator topology, and uniformly for $\zeta$ in any compact subset of $\Lambda$, and equals $T_{V}^{\zeta}$. Finally: if $\phi$ is any integrable function on the real line, then

$$
\lim _{|\tau| \rightarrow 0} \int_{-\infty}^{+\infty}\left(T_{V, \tau}^{i s} f, g\right) \phi(s) d s=\int_{-\infty}^{+\infty}\left(T_{V}^{i s} f, g\right) \phi(s) d s,
$$

for all $f, g$ in $\mathscr{L}_{2}$ (where $T_{V}^{i s}$ is the strongly continuous extension of $T_{V}^{\ell}$ to the imaginary axis).

REMARK 5.1. The fact of convergence was proved by D. Babbitt [1], under the added assumption that $V$ satisfied a local Lipschitz condition. The proof of the present generalization is just a simplification of Babbitt's proof.

Proof of theorem. Consider the sum $\sum_{j} V\left(\xi_{\left(\tau_{1}+\ldots+\tau_{j}\right) t}(\omega)\right) \tau_{j} t$. This is a Riemann sum for the integral $\int_{0}^{t} V\left(\xi_{s}(\omega)\right) d s$, using the partition $\left(\tau_{1} t, \cdots, \tau_{n(\tau)} t\right)$. Thus, $\sum_{j} V\left(\xi_{\left(\tau_{1}+\ldots+\tau_{j}\right) t}(\omega)\right) \tau_{j} t$ converges to $\int_{0}^{t} V\left(\xi_{s}(\omega)\right) d s$ as $|\tau| \rightarrow 0$, for $\operatorname{Pr}_{x}$-almost every $\omega$. Now let $f$ be in $\mathscr{M}$. Then, since the functions within $E_{x}\{\ldots\}$ are all bounded in norm by $\left|f\left(\xi_{t}\right)\right|$, and converge $\operatorname{Pr}_{x}-$ a.e., we have

for each $x$. Also

$$
\lim _{|\tau| \rightarrow 0} E_{x}\left\{\exp \left[-\sum_{j} V\left(\xi_{\left.\zeta \tau_{1}+\ldots+\tau_{j}\right) t}\right) \tau_{j} t\right] f\left(\xi_{t}\right)\right\}=T_{V}^{t} f(x)
$$

$$
\left|E_{x}\left\{\exp \left[-\sum_{j} V\left(\xi_{\left(\tau_{1}+\ldots+\tau_{j}\right) t}\right) \tau_{j} t\right] f\left(\xi_{t}\right)\right\}-T_{V}^{t} f(x)\right|^{2} \leqq 2 E_{x}\left\{\left|f\left(\xi_{t}\right)\right|\right\}^{2} .
$$

Thus, if $f$ is in $\mathscr{L}_{2}$, then $\lim _{|\tau| \rightarrow 0}\left\|T_{V, \tau}^{t} f-T_{V}^{t} f\right\|_{2}=0$, i.e. $T_{V, \tau}^{t}$ converges strongly to $T_{V}^{t}$. Now we can apply Fact 4.1 to get the existence of a holomorphic limit $T_{V}^{\zeta}, \zeta$ in $\Lambda$, which must agree with $e^{-\zeta A V}$ on $\bar{\Lambda}$ since it agrees for $\zeta>0$.

The fact that $\lim _{|\tau| \rightarrow 0} \int_{-\infty}^{\infty}\left(T_{V, \tau}^{i s} f, g\right) \phi(s) d s=\int_{-\infty}^{\infty}\left(T_{V}^{i s} f, g\right) \phi(s) d s$ for all $\phi$ in $\mathscr{L}_{1}$ is a consequence of the fact that $\left(T_{V, \tau}^{\zeta} f, g\right)$ and $\left(T_{V}^{\zeta} f, g\right)$ are bounded holomorphic functions and $\left(T_{V, \tau}^{\zeta} f, g\right) \rightarrow\left(T_{V}^{\zeta} f, g\right)$ on $\Lambda$. This can be seen as follows. Let $P_{t}(s)=(1 / \pi)\left(t /\left(t^{2}+s^{2}\right)\right) . P_{t}$ is an approximate identity, so that $P_{t} * \phi \rightarrow \phi$ in $\mathscr{L}_{1}(-\infty, \infty)$. If $\Psi$ is any bounded analytic function in the right half plane, then $\Psi(t+i s)=\int P_{t}\left(s-s^{\prime}\right) \Psi\left(i s^{\prime}\right) d s^{\prime}$. Now let $\Psi_{\tau}(\zeta)=\left(T_{V, \tau}^{\zeta} f, g\right)$, and $\Psi(\zeta)=\left(T_{V}^{\zeta} f, g\right)$. Notice that $\int P_{t} * \psi(s) \phi(s) d s=\int \psi(s) P_{t} * \phi(s) d s$. Thus: $\int\left(\Psi_{\tau}(i s)-\Psi(i s)\right) \phi(s) d s$ $=\int\left(\Psi_{\tau}(t+i s)-\Psi(t+i s)\right) \phi(s) d s+\int\left(\Psi_{\tau}(i s)-\Psi(i s)\right)\left(\phi(s)-P_{t} * \phi(s)\right) d s$. The second term has absolute value $\leqq$ const. $\int\left|\phi(s)-P_{t} * \phi(s)\right| d s$. By choosing $t$ small, this can be made arbitrarily small (for fixed $\phi$ ). The first term can then be made small by choosing $|\tau|$ small, since $\left|\Psi_{\tau}(t+i s)-\Psi_{\tau}(t+i s)\right|$ stays bounded by $2\|f\|\|g\|$, and converges to 0 for each $s$.

REMARK 5.2. Observe that one point which came out in the proof was that for each $x$ at which $V$ is Riemann-approximable, 


$$
E_{x}\left\{\exp \left[-\sum_{j} V\left(\xi_{\left(\tau_{1}+\ldots+\tau_{j}\right) t}\right) \tau_{j} t\right] f\left(\xi_{t}\right)\right\}
$$

converges to $T_{V}^{t} f(x)$, for each $f$ in $\mathscr{M}$. (Note: Riemann-approximabity at $x$ has not been defined, but it should be obvious what is meant.)

What sort of $V$ are Riemann-approximable? A large class is the following. It permits arbitrarily bad infinities on a set of capacity zero.

THEOREM 5.2. Let $D$ be the closed set of $x$ for which $V$ is essentially unbounded in every neighborhood of $x$. Suppose D forms a set of capacity 0 . Suppose also the points of discontinuity of $V$ form a set of measure 0 . Then $V$ is Riemann-approximable.

Proof. By changing $V$ on a set of measure 0 in $D^{\perp}$, we can assume that $V$ is actually locally bounded in $D^{\perp}$. Namely, let $C_{n} \uparrow D, C_{n}$ compact, and replace $V$ on $C_{n}-C_{n-1}$ by $V \wedge\left\|1_{C_{n}} V\right\|$. This will not introduce any new discontinuities. Then $\operatorname{Pr}_{x}\left\{\xi_{s}\right.$ lies in $D$ for some $\left.s\right\}=0$, for a set $D$ of capacity 0 . See, for example, [4]. Thus, for $\operatorname{Pr}_{x}$-a.e. $\omega$, the range of $\xi_{s}(\omega), 0 \leqq s \leqq t$, is a compact subset of $D^{\perp}$, and so $s \rightarrow V\left(\xi_{s}(\omega)\right)$ is bounded on $[0, t]$. Also, for $\operatorname{Pr}_{x}$-a.e. $\omega$, the set of $s$ for which $\xi_{s}(\omega)$ lies in the set of discontinities of $V$ has Lebesgue measure 0 . Thus, for $\operatorname{Pr}_{x}$-a.e. $\omega, \xi_{s}(\omega)$ is Riemann-integrable for $0<s \leqq t$.

6. The Green's function. Recall that if $f \in \mathscr{L}_{p}$ then $\left\|T_{V}^{t} f\right\|_{\infty} \leqq C(q) t^{(1 / q-1)^{k} / 2}$. So, for $1 \leqq p<\infty, T_{V}^{t} f(x)=\int k_{x}^{t}(y) f(y) d y$, where $k_{x}$ is an equivalence class of Lebesgue measurable functions, and $\left\|k_{x}^{t}\right\|_{q} \leqq C(q) t^{(1 / q-1)^{k} / 2}$. For $f \geqq 0$ in $\mathscr{L}_{\infty}$ choose $f_{n} \in \mathscr{L}_{2}, f_{n} \uparrow f$. Then $T_{V}^{t} f_{n}(x) \uparrow T_{V}^{t} f(x)$, so that

$$
\int k_{x}^{t}(y) f(y) d y=\lim _{n \rightarrow \infty} \int k_{x}^{t} f_{n}(y) d y=\lim _{n \rightarrow \infty} T_{V}^{t} f_{n}(x)=T_{V}^{t} f(x),
$$

so again we have $\int k_{x}^{t}(y) f(y) d y=T_{V}^{t} f(x),\left\|k_{x}^{t}\right\|_{1} \leqq C(1)$, independent of $t$.

We introduce a canonical version of $k_{x}^{t}$.

LEMMA 6.1. $\int k_{x}^{r}(z) k_{y}^{s}(z) d z$ is, for each $x$, equal to $k_{x}^{t}(y)$ for almost every $y$, provided $r+s=t$. Further, it is independent on the choice of $r$ and $s$.

Proof. $k_{x}^{s}(y)$ can be chosen a jointly Borel measurable function of $x$ and $y$, since the map $x \rightarrow k_{x}^{s}$ is a measurable map from $\mathscr{K}$ to, for example, $\mathscr{L}_{2}$. Furthermore,

$$
\int g(x) k_{x}^{s}(y) f(y) d y=\int g(x) T_{V}^{s} f(x) d x=\int T_{V}^{s} g(y) f(y) d y,
$$

since $T_{V}^{s}$ is self-adjoint. So

$$
\begin{aligned}
\int\left(\int k_{x}^{r}(z) k_{z}^{s}(y) d z\right) f(y) d y & =\iint k_{x}^{r}(z) k_{z}^{s}(y) f(y) d y d z=\int k_{x}^{r}(z) T_{V}^{s} f(z) d z=T_{V}^{r} T_{V}^{s} f(x) \\
& =\int k_{x}^{t}(y) f(y) d y .
\end{aligned}
$$


To show independence of $r$ and $s$, we choose $r, s, r^{\prime}, s^{\prime}$, with $r+s=r^{\prime}+s^{\prime}$. Assume $r<r^{\prime}$. Then, from what has been shown,

$$
\begin{aligned}
\int k_{x}^{r^{\prime}}(z) k_{y}^{s^{\prime}}(z) d z & =\int k_{x}^{r+\left(r^{\prime}-r\right)}(z) k_{y}^{s^{\prime}}(z) d z \\
& =\iint k_{x}^{r}(q) k_{w}^{r^{\prime}-r}(z) k_{z}^{s^{\prime}}(y) d w d z \\
& =\iint k_{x}^{r}(w) k_{z}^{s-s^{\prime}}(w) k_{z}^{s^{\prime \prime}}(y) d w d z \\
& =\int k_{x}^{r}(w) k_{w}^{s}(y) d w
\end{aligned}
$$

which completes the proof.

Now it makes sense to define $K_{V}^{t}(x, y)=\int k_{x}^{r}(z) k_{y}^{s}(z) d z$, since it is independent of $r$ and $s$, provided $r+s=t$.

Thus we have

REMARK 6.1. (a)There is a function $K_{V}^{t}(x, y)$ such that $T_{V}^{t} f(x)=\int K_{V}^{t}(x, y) f(y) d y$ for $f$ in any $\mathscr{L}_{p}$-class. $K_{V}^{t}$ is symmetric. Further, $K_{V}^{s+t}(x, z)=\int K_{V}(x, y) K_{V}(y, z) d y$.

(b) The last property, together with the fact that $T_{V}^{t} f(x)=\int K_{V}^{t}(x, y) f(y) d y$ for enough $f$, uniquely determine $K_{V}^{t}$. (I use the label "remark" rather than "theorem" in order to avoid being precise about the word "enough".)

Properties of $T_{V}^{t}$ easily translate into properties of $K_{V}^{t}$. For example:

THEOREM 6.1. $x \rightarrow K_{V}^{t}(x, \cdot)$ is continuous into all $\mathscr{L}_{p}$, and $K_{V}^{t}$ is jointly continuous in $x$ and $y$, on the open set where $V$ is locally integrable.

Proof. The first statement is an immediate consequence of the definition and of Theorem 3.5. As for the second part: if $x_{n} \rightarrow x$ and $y_{n} \rightarrow y$, and $V$ is locally integrable at $x$ and $y$, then

$$
\begin{aligned}
& \left|\int K_{V}^{r}\left(x_{n}, z\right) K_{V}^{s}\left(y_{n}, z\right) d z-\int K_{V}^{r}(x, z) K_{V}^{s}(y, z) d z\right| \\
& \quad \leqq\left\|K_{V}^{r}\left(x_{n}, \cdot\right)-K_{V}^{r}(x, \cdot)\right\|_{2}\left\|K_{V}^{s}\left(y_{n}, \cdot\right)\right\|_{2}+\left\|K_{V}^{r}(x, \cdot)\right\|_{2}\left\|K_{V}^{s}\left(y_{n}, \cdot\right)-K_{V}^{s}\left(y_{n}, \cdot\right)\right\|_{2} \cdot
\end{aligned}
$$

But $\left\|K_{V}^{s}\left(y_{n}, \cdot\right)\right\|_{2}$ and $\left\|K_{V}^{r}\left(x_{n}, \cdot\right)\right\|_{2}$ stay bounded, while the other factors go to zero.

\section{REFERENCES}

1. D. G. Babbitt, A summation procedure for certain Feynman integrals, Doctoral dissertation, Univ. of Michigan, 1962. See also Abstract 62T-298, Notices Amer. Math. Soc. 9 (1962), 402.

2. S. G. Brush, Functional integrals and statistical physics, U.S. Atomic Energy Comm., Tech. Rep. No. UCRL-5694-T, (1959).

3. R. H. Cameron, A family of integrals serving to connect the Wiener and Feynman integrals J. Math. and Phys. 39 (1960), 126-140. 
4. J. L. Doob, Semimartingales and subharmonic functions, Trans. Amer. Math. Soc. 77 (1954), 86-121.

5. E. B. Dynkin, On some transformations of Markoff processes, Dokl. Akad. Nauk. SSSR 133 (1960), 269-272.

6. R. J. Feynman, Space-time approach to nonrelativistic quantum mechanics, Rev. Modern Phys. 20 (1948), 367-387.

7. I. M. Gel'fand and A. M. Yaglom, Integration in function space and its applications in quantum physics, J. Mathematical Phys. 1 (1960), 48-69.

8. R. Getoor, Additive functionals of a Markov process, Pacific J. Math. 7 (1957), 1577-1591.

9. —_ Markov operators and their associated semi-groups, Pacific J. Math. 9 (1959), $449-472$.

10. E. Hille and R. S. Phillips, Functional analysis and semigroups, Amer. Math. Soc. Colloq. Publ. Vol. 31, rev. ed., Amer. Math. Soc., Providence, R.I., 1957.

11. M. Kac, On some connections between probability theory and differential and integral equations, Proc. 2nd Berkeley Symposium on Math. Statist. and Prob., Univ. of California Press, Berkeley, Calif., 1951.

12. E. W. Montroll, Markov chains, Wiener integrals and quantum theory, Comm. Pure Appl. Math. 5 (1952), 415-453.

13. B. Sz.-Nagy, Spektraldarstellung linearer Transformationen des Hilbertischen Raumes, Springer-Verlag, Berlin, 1942.

14. E. Nelson, N. R. S. Colloques sur les équations aux dérivees partielles, Paris, 1962.

15. D. Ray, On the spectra of second order differential operators, Trans. Amer. Math. Soc. 77 (1954), 299-321.

16. M. Rosenblatt, On a class of Markov processes, Trans. Amer. Math. Soc. 71 (1951), 120-135.

17. V. A. Volkonskii, Additive functionals of Markov processes, Trudy Moskov. Mat. Obšč. 9 (1960), 143-189. (Russian)

\section{UNIVERSTTY of CALIFornia, \\ Berkeley, California}

